BMJ Open Sport \& Exercise Medicine

\section{Positioning of the femoral tunnel in anterior cruciate ligament reconstruction: functional anatomical reconstruction}

To cite: Jorge PB, Escudeiro D, Severino NR, et al. Positioning of the femoral tunnel in anterior cruciate ligament reconstruction: functional anatomical reconstruction. $B M J$ Open Sport \& Exercise Medicine 2018:4:e000420. doi:10.1136/ bmjsem-2018-000420

Accepted 29 August 2018

\section{Check for updates}

(c) Author(s) (or their employer(s)) 2018. Re-use permitted under CC BY-NC. No commercial re-use. See rights and permissions. Published by BMJ.

Departamento de Ortopedia e Traumatologia da, Irmandade da Santa Casa de Misericórdia de, São Paulo, Brazil

Correspondence to Dr Diego Escudeiro; diegoescudeiro@gmail.com

\section{ABSTRACT}

The aim of this study was to review and update the literature in regard to the anatomy of the femoral origin of the ACL, the concept of the double band and its respective mechanical functions, and the concept of direct and indirect fibres in the ACL insertion. These topics will be used to help determine which might be the best place to position the femoral tunnel and how this should be achieved, based on the idea of functional positioning, that is, where the most important ACL fibres in terms of knee stability are positioned. Low positioning of the femoral tunnel, reproducing more of the posterolateral band, and positioning the tunnel away from the lateral intercondylar ridge, that is, in the indirect fibres, would theoretically rebuild a ligament that is less effective in relation to knee stability. The techniques described to determine the femoral tunnel's centre point all involve some degree of subjectivity; the point is defined manually and depends on the surgeon's expertise. The centre of the ACL insertion in the femur should be used as a parameter. Once the centre of the ligament in its footprint is marked, the centre of the tunnel must be defined, drawing the marking toward the intercondylar ridge and anteromedial band. This will allow the femoral tunnel to occupy the region containing the most important original ACL fibres in terms of this ligament's function.

\section{INTRODUCTION}

The ACL is one of the knee's most frequently injured structures. At least one in every nine patients undergoing reconstruction of this ligament suffers re-rupture or clinical failure (traumatic or atraumatic) in the long term. ${ }^{12}$ For this reason, detailed knowledge of the ACL's anatomical characteristics is a basic prerequisite for any orthopaedic surgeon. ${ }^{23}$ A multicentre study of ACL revisions (MARS Group-Multicenter ACL Revision Study) ${ }^{4}$ produced a data sample of more than 1000 revision surgeries for this ligament and reported that technical errors are the leading cause of surgical reconstruction failure. Most of this is due to poor positioning of the
What is already known

- Technical errors are the leading cause of surgica reconstruction failure. Most of this is due to poor positioning of the femoral tunnel (approximately 50\% of cases).

- The ACL occupies $13 \%$ of the medial wall of the lateral condyle, but anatomical variations in individual patients must always be considered.

- The direct type of ligament insertion involves a firm and fixed enthesis that allows a gradual load distribution on the subchondral bone and, from a biomechanical point of view, is extremely important as a key connection between ligament and bone for transmitting a mechanical load to the joint.

\section{What are the new discoveries}

With the increasing importance of the supposed anteromedial band and direct fibers, we believe that a tunnel position occupying a larger anteromedia area of the ACL origin and closer to the lateral femoral intercondylar ridge would be more efficient and would decrease the chance of positioning the tunnel to occupy a larger area of the posterolateral region.

- Reconstruction of the ACL should be understood as an individualised surgery, with large anatomical differences between patients. The concept of functional anatomical reconstruction is of great value because as the diameter of the neoligament insertion is less than the diameter of the original ligament, this technique seeks to reconstruct the ACL at its most important, most functional portion and not just at its anatomically most central portion.

femoral tunnel (approximately $50 \%$ of cases). The proportion of femoral, as opposed to tibial, tunnel positioning errors is $3: 1$ because the knee's centre of rotation is closer to the femoral insertion, and preparation of this tunnel is considered to be one of the most complex procedures in ACL reconstruction. ${ }^{1}$

The anatomy of the ACL has been studied for decades, ${ }^{5-8}$ and there are reports of 
reconstruction surgery since 1917, when it was performed by Hey Groves. ${ }^{9}$ Naturally, the anatomical concepts and surgical techniques have changed over the 20th and 21 st centuries, and even after 100 years of reconstructive surgery with satisfactory results demonstrated in various studies, there are still discussions about the anatomical aspects of the ACL and graft positioning in tunnels, especially the femoral tunnel. ${ }^{10-13}$ The concept of anatomical ACL reconstruction, in which the graft is placed in the native ACL insertion area, has developed in recent decades. This is in part due to renewed interest in the anatomy of the ligament insertion, using surgical techniques that are able to reproduce this anatomy reliably and precisely during surgical reconstruction. ${ }^{14}$ However, the question of whether the wide variety in the size and shape of the ACL's femoral origin may be a cause of the observed differences remains unclear. ${ }^{14}$

It is therefore evident that a more detailed description of the ACL's anatomy is needed in an attempt to replicate the anatomy and the exact behaviour of the ligament. ${ }^{15}$ Thus, the aim of this study was to review and update the literature in regard to the anatomy of the ACL's femoral origin, the concept of the double band and its respective mechanical functions, and the concept of direct and indirect fibres in ACL insertion. Based on these topics, the goal is to determine the best location to position the femoral tunnel and how to achieve it, as the tunnel to be performed is smaller and geometrically distinct from the area occupied by the native ACL. The idea is to position the new ligament in the region where the native ACL fibres play the greatest role in terms of anteroposterior and rotational stability.

Two independent authors performed the search and selection of studies in the MEDLINE (via PubMed), Cochrane Library and LILACS databases, using the following terms: 'anterior cruciate ligament', 'anterior cruciate ligament reconstruction', 'anatomy', 'footprint', 'tunnel', 'femur' and 'femoral'. The search was conducted in December 2017. There were no date restrictions. Studies analysing the ACL's anatomy and biomechanics and those evaluating the positioning of the femoral tunnel were included. Experimental studies and those published in languages other than English or Portuguese were excluded. Analysis of the studies was presented in a descriptive way, didactically separated into topics according to the objectives of this study.

\section{Anatomy of the ACL}

The ACL is an intracapsular and extrasinovial structure. It is the main static stabiliser in the anterior translation of the tibia relative to the femur and helps the restriction of external rotation of the tibia. ${ }^{13}$ It is known that the femoral origin and tibial insertion of the ACL, as well as the different shapes and diameters along the length of the ligament, range from knee to knee. This makes it difficult to define where the tunnel should be positioned during surgical reconstruction. ${ }^{16}$ The ACL has an average length of $32 \mathrm{~mm}$ (range, 22 to $41 \mathrm{~mm}$ ). It is inserted laterally and anteriorly to the tibia's medial intercondylar spine and in the medial wall of the lateral femoral condyle. ${ }^{17}$ Its width ranges from 7 to $17 \mathrm{~mm}$ in diameter, with an average of $11 \mathrm{~mm} .{ }^{3}$ Its footprint on the medial surface of the lateral femoral condyle is egg-shaped and oriented to approximately 30 degrees of flexion when compared with the long axis of the femur. ${ }^{18}$ Its area ranges from 93 to $200 \mathrm{~mm}^{2} .{ }^{19}$

A cadaver study ${ }^{15}$ showed that on average, the ACL occupies $13 \%$ of the medial wall of the lateral condyle, but anatomical variations in individual patients must always be considered.

Given these facts, it is clear that reproduction of the ACL's complex anatomy with existing reconstruction techniques is practically impossible, and what is sought is a reconstruction that is as close as possible to the original anatomy. ${ }^{15}$

\section{Anteromedial and posterolateral bands}

Reporting of the presence of two bands within the ACL began in $1938,{ }^{20}$ and in 1975 , a division of the ligament into two parts with different functions was observed. In 2007, the presence of two well-characterised bands was demonstrated in fetuses. ${ }^{3}$ The two bands differ in length, the anteromedial (AM) band being longer than the posterolateral (PL). When viewed arthroscopically at 90 degrees of knee flexion, the two bands are positioned almost horizontally, the PL band anterior to the AM. In full extension, the bands come together almost vertically, with the AM band proximal to the PL. The femoral insertions of the AM and PL bands are often (in approximately $80 \%$ of subjects) separated by a bony landmark, the bifurcated lateral ridge. Between the femoral origin and tibial insertion, the bands are positioned in parallel in extension and twisted at 90 degrees of knee flexion. Both bands resist anterior translation and external rotation of the tibia relative to the femur, with the AM band more tense from 30 degrees to 90 degrees, and the PL from 0 degrees to 30 degrees of knee flexion. ${ }^{17}$

Two similar studies published in $2013^{21}$ and $2015^{22}$ evaluated the mechanical functions of the AM and PL bands. Both studies analysed four models in cadaver knees: (1) normal knee, (2) knee with resected AM band, (3) knee with resected PL band and (4) knee with completely resected ACL. These knees were evaluated mechanically in order to compare rotational and anterior-posterior instability of the tibia in relation to the femur. Both authors found that the AM band had greater anteroposterior and rotational stability. These studies showed the superiority of the AM band in relation to the PL band, and knowledge of this fact is essential when choosing femoral tunnel positioning during ACL reconstruction.

Despite these descriptions, it has been argued that the ACL has a tape format, rather than two bands, and in reality, what occurs is that this double band phenomenon occurs when flexing the knee (figure 2), that is, there appears to be two bands, when in reality there is only one. ${ }^{7}$ This has been a recent discussion over the past 
decade and may affect the choice of graft and also the determination of the ideal place for performing femoral and tibial tunnels.

\section{Direct and indirect fibers}

Ligaments have two modes of insertion into the bone, known as direct and indirect insertion. Direct insertion has a zonal architecture, including the transition of ligament tissue, non-calcified cartilage and cartilage calcified to bone, which allows a progressive distribution of force and differential tension of all insertion components at the insertion site. Indirect insertion has a significantly simpler ultrastructure, where the ligament is anchored directly to the bone by collagen fibres without forming a clear transition zone. ${ }^{21}$

The direct type of ligament insertion involves a firm and fixed enthesis that allows a gradual load distribution on the subchondral bone and, from a biomechanical point of view, is extremely important as a key connexion between ligament and bone for transmitting a mechanical load to the joint. Indirect insertion is an indirect anchoring of ligament to bone and is of secondary importance in ligament function. ${ }^{23}$

A study published in $2010^{24}$ divided ACL insertion into direct and indirect fibres. In 2012, another histological study ${ }^{25}$ noted a microscopic structure of four layers in both the femoral and tibial direct insertion, demonstrating that direct fibres are stronger and biomechanically more important, whereas indirect fibres work only as ligament anchor points. In 2014, another study was published in which the authors reported difficulty in reconstructing indirect fibres when using tunnels. However, it was observed that it is possible to recreate the remaining ACL substance fibres with the currently used techniques. ${ }^{26}$

The positioning described in the following paragraphs relates to the arthroscopic view, where anterior means anatomically inferior, posterior means superior, low means posterior and high means anterior.

Direct ACL insertion is located anteriorly, on the medial surface of the lateral femoral condyle. It is connected to a bone depression parallel and immediately posterior to the intercondylar lateral ridge and to the medial surface of the lateral femoral condyle. It is suggested that the direct insertion fibre architecture is an important stabilising structure, consisting of a band of dense collagen fibres with a zonal architecture that moves from the ligament to the fibrocartilage, to the mineralised fibrocartilage and then to the bone. ${ }^{27}$ It is a resistant and firm insertion from a biomechanical point of view, as this tissue transition provides the load distribution in a gradual manner. ${ }^{19}$

Indirect ACL insertion is represented by the most posterior femoral insertion fibres and is fan-shaped, bordering the articular cartilage when the knee is fully extended. ${ }^{26}$

The fibres inserted in areas that correspond to an AM band provide $66 \%$ to $84 \%$ of the total resistance to the anterior drawer from 0 degrees to 90 degrees of flexion.
The contribution of the fibres comprising the PL portion of the band decreases to $16 \%$ at 0 degrees and $9 \%$ at 90 degrees. ${ }^{28}$ The superior fibres of the ACL femoral insertion (anterior band), adjacent to the intercondylar lateral ridge, possess greater strength than the inferior fibres in relation to the Lachman, anterior drawer and pivot shift simulated tests. Furthermore, the virtual fibres located in the superior AM portion of the femoral ACL insertion are more isometric than the fibres inserted in the inferior region. Given this fact, it could be advantageous to create a 'superior' femoral tunnel during ACL reconstruction, in the lateral intercondylar ridge. ${ }^{29}$

Data from a 2016 study and from the previously summarised literature indicate that the high fibres of the femoral footprint of the surrounding ACL and on the lateral intercondylar ridge (1) exert greater force during the Lachman and pivot shift tests, (2) play a dominant role in the control of translations and rotations during the Lachman and pivot shift tests, and (3) represent the most isometric region of the footprint. ${ }^{27}$

There are results that do not support low positioning of the femoral tunnel, as this region of the footprint supports less load than the upper region. In fact, the tendency to perform 'lower' femoral tunnels can be harmful to the graft, as recent biomechanical studies have shown greater changes in length and resulting strength in low anatomical grafts during range of motion. ${ }^{27}$

A limitation of the above listed studies is that they were performed using cadaver knees of advanced age. There is a lack of clinical studies with young patients' knees that could confirm that these histological characteristics exist similarly in young people. ${ }^{28}$

\section{Current anatomical reconstruction}

There is no consensus on the anatomical positioning of the femoral tunnel, and the anatomical centre of the native femoral insertion is primarily taken into account. Van Eck et $a l^{30}$ recommended that when determining ACL reconstruction, one should consider positioning the tunnel at the centre of the native one and also the orientation of the collagen fibres and insertion sites.

A systematic review published by Piefer $e t a l^{31}$ in 2011 showed that the anatomical centre of the femoral ACL origin was $43 \%$ of the proximal to distal length of the articular edge. $\mathrm{Xu}$ et $a l^{1}$ also performed a systematic review with the aim of establishing the normal position of the femoral ACL origin and demonstrated the probable practical location of this point during arthroscopy and the postoperative evaluation method. The position of the femoral tunnel was evaluated according to the quadrants method described by Bernard et $a l,{ }^{32}$ and it was possible to observe among the 13 studies included in this review that, in theory, the ACL centre as a whole was $28.4 \%$ of the total diameter of the femoral condyle along the Blumensaat line and $35.7 \%$ of the maximum height of the intercondylar ridge, whereas the AM band was $24.2 \%$ of the distance from the centre of the femoral origin to the medial edge and $21.6 \%$ of the distance from the 


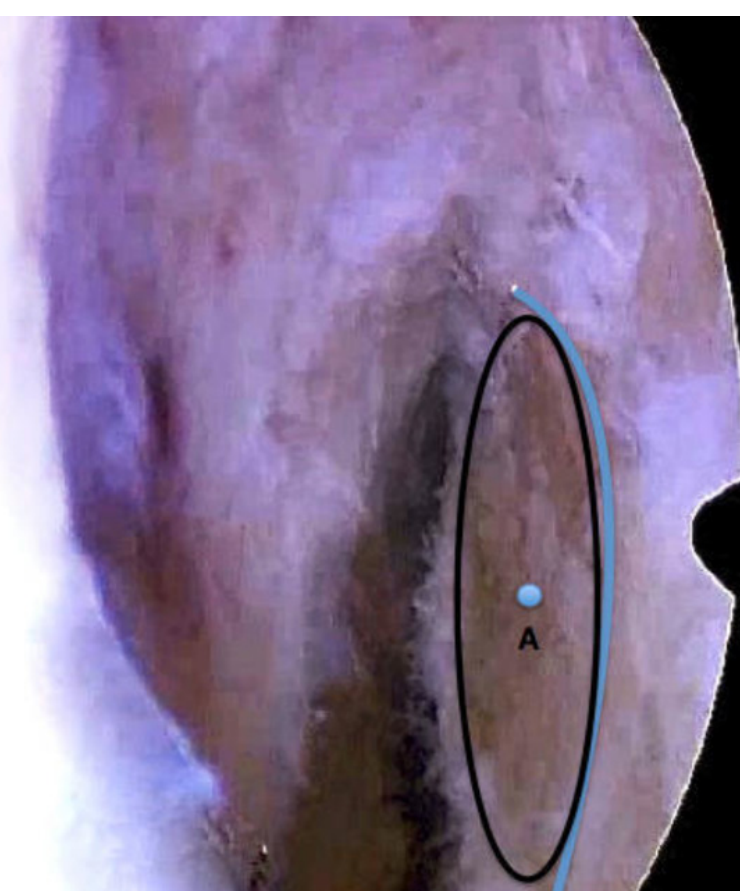

Figure 1 Point A: the centre of the $A C L$ is demarcated with wire or an 'ice pick', and we thus obtain the anatomical positioning already described in previous articles. ${ }^{1-3}$

centre to the Blumensaat line; the PL band was $32.8 \%$ and $46.7 \%$ of these distances, respectively. By combining the data, the authors showed that the anatomical centre of the femoral ACL origin from the proximal to the distal articular margin was $50.8 \%$, on a line parallel to the femoral anatomical axis.

The techniques described to determine the femoral tunnel's centre point all involve some degree of subjectivity; the point is defined manually and depends on the surgeon's expertise. In addition, the position of the new tunnel in the central region of the old ligament should be taken into account. The authors propose that the tunnel should be inserted within the ACL's footprint in a region where it matters most functionally: functional anatomical positioning.

\section{Where should the femoral tunnel be positioned?}

In the opinion of the authors of this article, the centre of the ACL origin should be used as a parameter, using the remaining fibres of the lateral femoral intercondylar ridge and bifurcated wall, when present. Having marked the centre of the ACL, one must define the centre of the tunnel, taking the already marked ACL to a region closer to the intercondylar ridge and closer to the AM band than the PL band.

After cleaning the inner wall of the lateral femoral condyle, one must identify the lateral intercondylar ridge and the entire region of the ACL origin, as shown in figure 1. At this time, the centre of the ACL is demarcated with wire or an 'ice pick', and we thus obtain the anatomical positioning already described in previous articles, ${ }^{1-3}$ which we call point $\mathrm{A}$. Based on this parameter, we draw an imaginary line perpendicular to the lateral

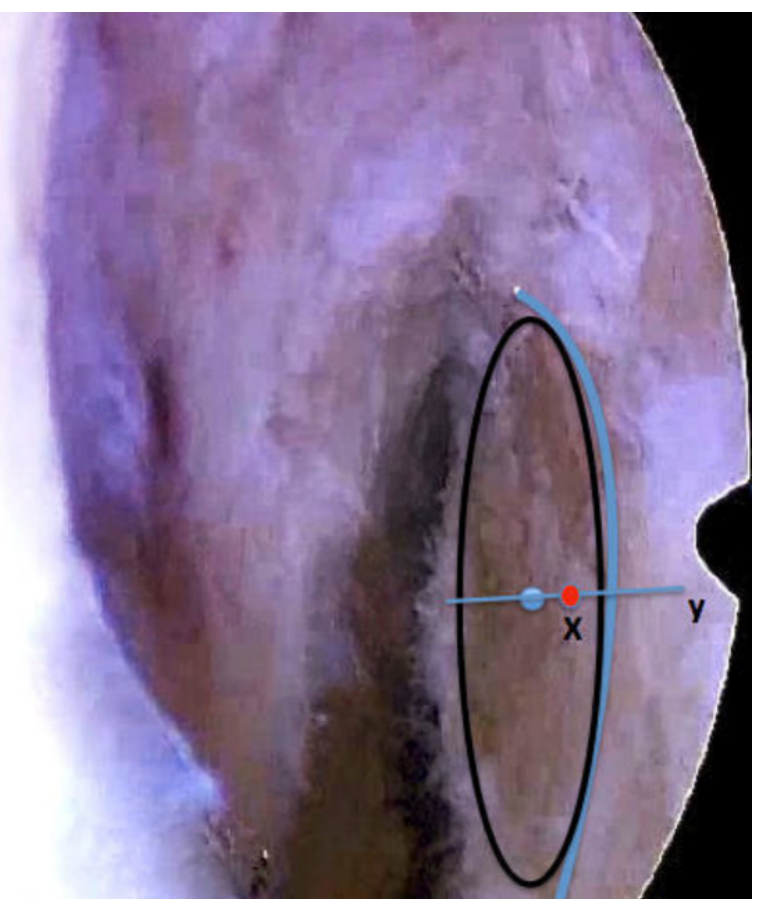

Figure 2 Line y: imaginary line perpendicular to the lateral intercondylar ridge. Point X: 1 to $2 \mathrm{~mm}$ in distance to point $\mathrm{A}$.

intercondylar ridge (line y) and take the central marking of the ACL (point A) towards the ridge, 1 to $2 \mathrm{~mm}$ in distance, using the area of both ends of the 'ice pick' as a parameter for this distance because it is approximately $1 \mathrm{~mm}$ in diameter. We call this new point X (figure 2). Then, we draw a new imaginary line (z) perpendicular to imaginary line $y$, in the direction of the anteromedial region of the ACL origin, and the centre of the tunnel is demarcated 1 to $2 \mathrm{~mm}$ from point $\mathrm{X}$, using the two ends

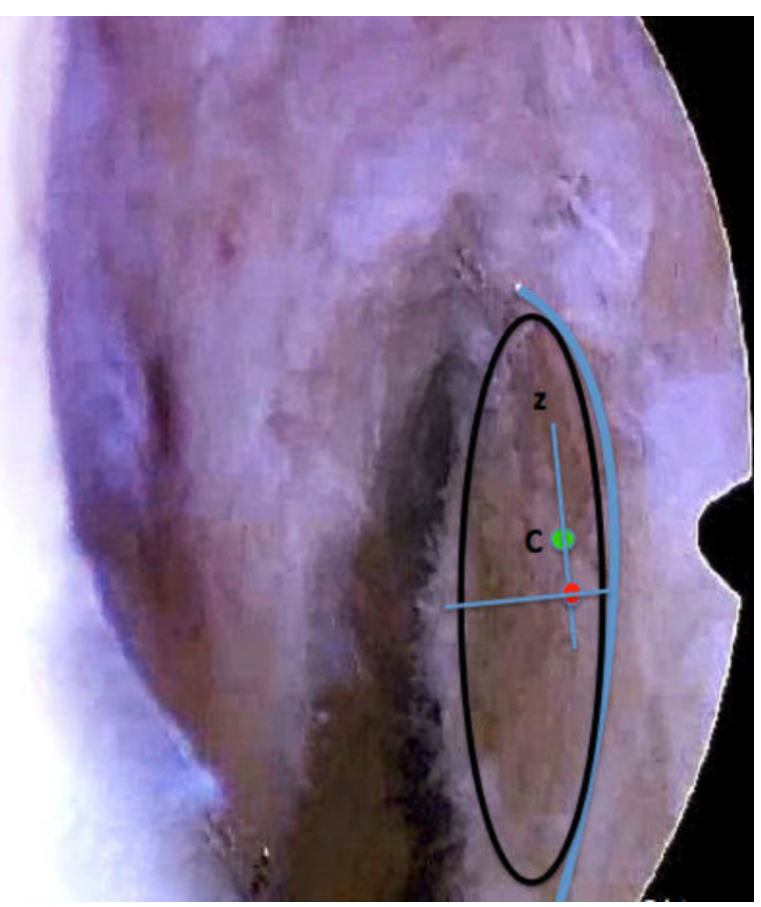

Figure 3 Point C: centre of the femoral tunnel to be made. 


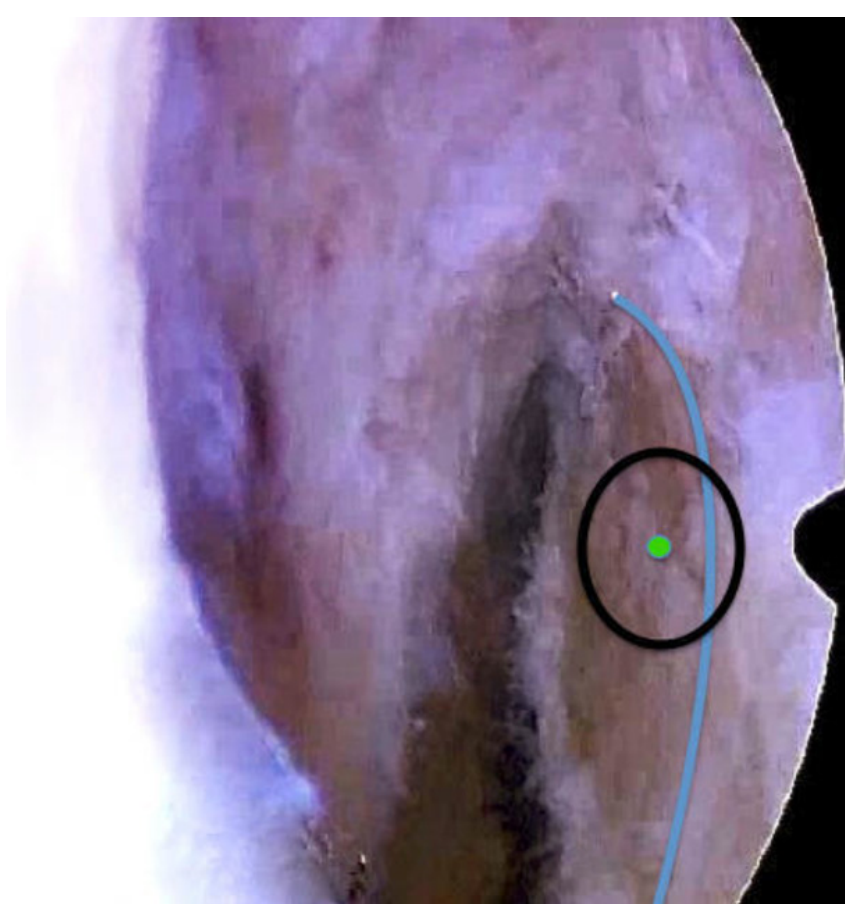

Figure 4 Tunnel representation.

of the 'ice pick' again as a parameter for this distance of 1 to $2 \mathrm{~mm}$, finally defining the centre of the femoral tunnel to be made (point $\mathrm{C}$ ) as shown in figure 3 . Figure 4 shows this demarcation being performed arthroscopically.

\section{CONCLUSION}

It is obvious and expected that there is a subjective aspect to performing the femoral tunnel, as there are anatomical variations from knee to knee and there is no customised guide for each type of joint. However, based on the literature discussed in this study, with the increasing importance of the supposed AM band and direct fibres, we believe that a tunnel position occupying a larger anteromedial area of the ACL origin and closer to the lateral femoral intercondylar ridge would be more efficient and would decrease the chance of positioning the tunnel to occupy a larger area of the posterolateral region, which theoretically predisposes it to a greater number of faults and failures.

The importance of adequate knowledge of the anatomy of the lateral femoral condyle's inner wall and careful and experienced viewing in terms of the correct understanding of anatomical parameters is clear, that is, we must look carefully at and understand the repair points on the condyle wall, which vary from individual to individual. Reconstruction of the ACL should be understood as an individualised surgery, with large anatomical differences between patients. The concept of functional anatomical reconstruction is of great value because as the diameter of the neoligament insertion is less than the diameter of the original ligament, this technique seeks to reconstruct the ACL at its most important, most functional portion and not just at its anatomically most central portion.
Acknowledgements All contributors meet the criteria for authorship.

Contributors All authors contributed to the design and implementation of the research, to the analysis of the results and to the writing of the manuscript.

Funding The authors have not declared a specific grant for this research from any funding agency in the public, commercial or not-for-profit sectors.

Competing interests None declared.

Patient consent Obtained.

Ethics approval Approval of Ethics Committee/Institutional Review Board is not necessary.

Provenance and peer review Not commissioned; externally peer reviewed.

Open access This is an open access article distributed in accordance with the Creative Commons Attribution Non Commercial (CC BY-NC 4.0) license, which permits others to distribute, remix, adapt, build upon this work non-commercially, and license their derivative works on different terms, provided the original work is properly cited, appropriate credit is given, any changes made indicated, and the use is non-commercial. See: http://creativecommons.org/licenses/by-nc/4.0/

\section{REFERENCES}

1. Xu H, Zhang C, Zhang Q, et al. A systematic review of anterior cruciate ligament femoral footprint location evaluated by quadrant method for single-bundle and double-bundle anatomic reconstruction. Arthroscopy 2016;32:1724-34.

2. Crawford SN, Waterman BR, Lubowitz JH. Long-term failure of anterior cruciate ligament reconstruction. Arthroscopy 2013;29:1566-71.

3. Ferretti M, Levicoff EA, Macpherson TA, et al. The fetal anterior cruciate ligament: an anatomic and histologic study. Arthroscopy 2007;23:278-83.

4. MARS Group, Wright RW, Huston LJ, et al. Descriptive epidemiology of the Multicenter ACL Study (MARS) cohort. Am J Sports Med 2010;38:1979-86.

5. Danylchuk KD, Finlay JB, Krcek JP. Microstructural organization of human and bovine cruciate ligaments. Clin Orthop Relat Res 1978;131:294-8.

6. Girgis FG, Marshall JL, Monajem A. The cruciate ligaments of the knee joint. Anatomical, functional and experimental analysis. Clin Orthop Relat Res 1975;106:216-31.

7. Smigielski R, Zdanowicz U, Drwięga M, et al. Ribbon like appearance of the midsubstance fibres of the anterior cruciate ligament close to its femoral insertion site: a cadaveric study including 111 knees. Knee Surg Sports Traumatol Arthrosc 2015;23:3143-50.

8. Amis AA, Dawkins GP. Functional anatomy of the anterior cruciate ligament. Fibre bundle actions related to ligament replacements and injuries. J Bone Joint Surg Br 1991;73:260-7.

9. Hey Groves E. Operation for the repair of the crucial ligaments. The Lancet 1917:190:674-6.

10. Harner CD, Marks PH, Fu FH, et al. Anterior cruciate ligament reconstruction: endoscopic versus two-incision technique. Arthroscopy 1994;10:502-12.

11. Sadoghi P, Müller PE, Jansson V, et al. Reconstruction of the anterior cruciate ligament: a clinical comparison of bone-patellar tendonbone single bundle versus semitendinosus and gracilis double bundle technique. Int Orthop 2011;35:127-33.

12. Koga $\mathrm{H}$, Muneta $\mathrm{T}$, Yagishita $\mathrm{K}$, et al. Mid- to long-term results of single-bundle versus double-bundle anterior cruciate ligament reconstruction: randomized controlled trial. Arthroscopy 2015;31:69-76.

13. Rayan F, Nanjayan SK, Quah C, et al. Review of evolution of tunnel position in anterior cruciate ligament reconstruction. World $J$ Orthop 2015;6:252-62.

14. Ferretti M, Ekdahl M, Shen W, et al. Osseous landmarks of the femoral attachment of the anterior cruciate ligament: an anatomic study. Arthroscopy 2007;23:1218-25.

15. Triantafyllidi E, Paschos NK, Goussia A, et al. The shape and the thickness of the anterior cruciate ligament along its length in relation to the posterior cruciate ligament: a cadaveric study. Arthroscopy 2013;29:1963-73.

16. Lee JK, Lee S, Seong SC, et al. Anatomy of the anterior cruciate ligament insertion sites: comparison of plain radiography and threedimensional computed tomographic imaging to anatomic dissection. Knee Surg Sports Traumatol Arthrosc 2015;23:2297-305.

17. Kopf S, Musahl V, Tashman S, et al. A systematic review of the femoral origin and tibial insertion morphology of the ACL. Knee Surg Sports Traumatol Arthrosc 2009;17:213-9. 
18. Yasuda $\mathrm{K}$, Kondo $\mathrm{E}$, Ichiyama $\mathrm{H}$, et al. Anatomic reconstruction of the anteromedial and posterolateral bundles of the anterior cruciate ligament using hamstring tendon grafts. Arthroscopy 2004;20:1015-25.

19. Pathare NP, Nicholas SJ, Colbrunn R, et al. Kinematic analysis of the indirect femoral insertion of the anterior cruciate ligament: implications for anatomic femoral tunnel placement. Arthroscopy 2014;30:1430-8.

20. Palmer I. On the injuries to the ligaments of the knee joint: a clinical study. 1938. Clin Orthop Relat Res 2007;454:17-22.

21. Komzák M, Hart R, Okál F, et al. AM bundle controls the anteriorposterior and rotational stability to a greater extent than the PL bundle-a cadaver study. Knee 2013;20:551-5.

22. Gardner EJ, Noyes FR, Jetter AW, et al. Effect of anteromedial and posterolateral anterior cruciate ligament bundles on resisting medial and lateral tibiofemoral compartment subluxations. Arthroscopy 2015;31:901-10.

23. Benjamin M, Moriggl B, Brenner E, et al. The "enthesis organ" concept: why enthesopathies may not present as focal insertional disorders. Arthritis Rheum 2004;50:3306-13.

24. Iwahashi T, Shino K, Nakata K, et al. Direct anterior cruciate ligament insertion to the femur assessed by histology and 3-dimensional volume-rendered computed tomography. Arthroscopy 2010;26:S13-S20.
25. Sasaki N, Ishibashi Y, Tsuda E, et al. The femoral insertion of the anterior cruciate ligament: discrepancy between macroscopic and histological observations. Arthroscopy 2012;28:1135-46.

26. Mochizuki T, Fujishiro H, Nimura A, et al. Anatomic and histologic analysis of the mid-substance and fan-like extension fibres of the anterior cruciate ligament during knee motion, with special reference to the femoral attachment. Knee Surg Sports Traumatol Arthrosc 2014;22:336-44.

27. Nawabi DH, Tucker S, Schafer KA, et al. ACL fibers near the lateral intercondylar ridge are the most load bearing during stability examinations and isometric through passive flexion. $A m$ J Sports Med 2016;44:2563-71.

28. Kawaguchi $\mathrm{Y}$, Kondo $\mathrm{E}$, Takeda $\mathrm{R}$, et al. The role of fibers in the femoral attachment of the anterior cruciate ligament in resisting tibial displacement. Arthroscopy 2015;31:435-44.

29. Siebold R. Flat ACL anatomy: fact no fiction. Knee Surg Sports Traumatol Arthrosc 2015;23:3133-5.

30. van Eck CF, Schreiber VM, Mejia HA, et al. "Anatomic" anterior cruciate ligament reconstruction: a systematic review of surgical techniques and reporting of surgical data. Arthroscopy 2010;26(9 Suppl):S2-S12.

31. Piefer JW, Pflugner TR, Hwang MD, et al. Anterior cruciate ligament femoral footprint anatomy: systematic review of the 21 st century literature. Arthroscopy 2012;28:872-81.

32. Bernard M, Hertel P, Hornung $\mathrm{H}$, et al. Femoral insertion of the ACL. Radiographic quadrant method. Am J Knee Surg 1997;10:14-21. 